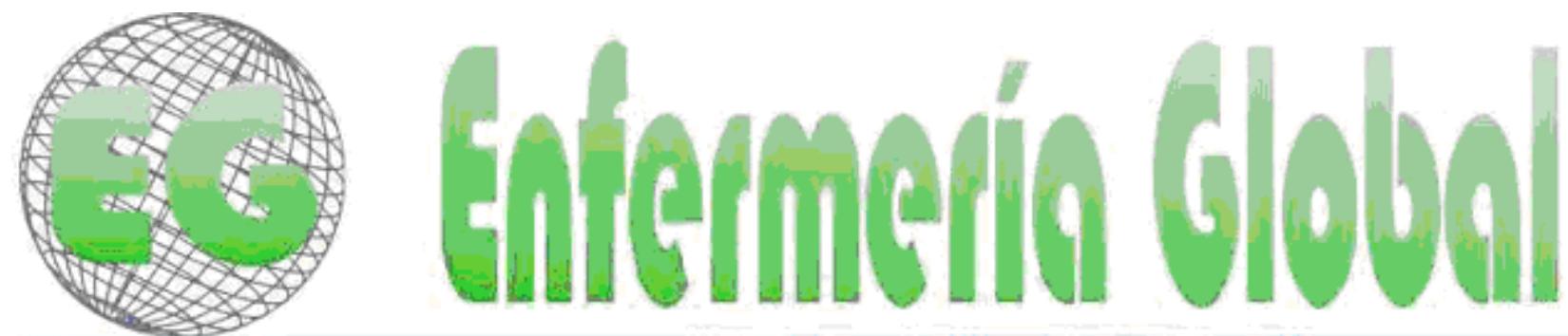

15 SN $1696-6+5 \%$

Revista electrónica cuatrimestral de Enfermeria

$\mathrm{N}^{\circ} 20$

Octubre 2010

www.um.es/eglobal/

\title{
CLÍNICA
}

\section{EXAMEN DE PAPANICOLAOU: FACTORES QUE INFLUYEN A LAS MUJERES A NO RECIBIR EL RESULTADO}

EXAME DE PAPANICOLAOU: FATORES QUE INFLUENCIAM AS MULHERES A NÃO RECEBEREM O RESULTADO

\author{
*Gomes Martins, L., **Bezerra Pinheiro, AK., *Moreira Vasconcelos, CT., \\ *Pinto Falcão Júnior, JS. \\ *Enfermera. **Doctora en Enfermería.Profesora Adjunta.Universidad Federal de Ceará - UFC. Brasil. \\ Palabras clave: Salud de la mujer; Neoplasmas del cuello uterino; Frotis vaginal \\ Palavras-chave: Saúde da mulher; Neoplasias do colo uterino; Esfregaço vaginal. \\ Keywords: Health of the woman; Cancer in the uterine neck; Smear vaginal
}

\section{RESUMEN}

El aumento en la prestación de servicios, prevención y tratamiento de casos de cáncer de cuello de útero tendrá limitado su ámbito de aplicación, si las mujeres no vuelven para recibir el resultado del examen de Papanicolaou. La no recepción de los informes citopatológicos acarrea discontinuidad del tratamiento, lo que impide terapias eficaces para las lesiones detectadas. Este estudio tiene como objetivo identificar las razones que llevan a las mujeres a no recibir los resultados de los exámenes de prevención. La encuesta se realizó con mujeres que han realizado su examen de prevención en el año 2005 hasta junio de 2006 y no volvieron para recibir el resultado. Un total de 29 mujeres colaboraron en la investigación. Los factores dificultadores para recibir el resultado del examen de Papanicoloau, según las mujeres entrevistadas, van desde cuestiones relacionadas con la organización de la institución a cuestiones relacionadas con la propia mujer. Sin embargo, las cuestiones relacionadas con la institución representan un porcentaje mucho mayor $(27,3 \%)$ en comparación con las demás. A partir de los factores que influyen en las mujeres a no recibir los informes, los profesionales podrán intervenir en la consulta haciendo hincapié en la importancia de recibir el resultado y tener un seguimiento regular.

\section{RESUMO}

O incremento na oferta de serviços, na prevenção e tratamento dos casos de câncer de colo uterino terá seu alcance limitado, se as mulheres não retornarem para receber o resultado do exame de Papanicolaou. O não recebimento dos laudos citopatológicos acarreta descontinuidade do tratamento, impedindo ações terapêuticas eficazes para as lesões detectadas. Este estudo teve 
como objetivo identificar os motivos que levam as mulheres a não receberem os resultados dos exames de prevenção. A pesquisa foi realizada com mulheres que fizeram seu exame de prevenção no ano de 2005 até junho de 2006 e não retornaram para receber o resultado. Um total de 29 mulheres colaboraram com a pesquisa. Os fatores dificultadores para o recebimento do resultado do exame de Papanicoloau, segundo as mulheres entrevistadas, perpassam desde questões ligadas à organização da instituição até questões ligadas à própria mulher. Todavia, as questões ligadas à instituição representaram uma parcela bem maior $(27,3 \%)$ quando comparada às demais. A partir dos fatores que influenciam as mulheres a não receberem os laudos, os profissionais poderão intervir durante a consulta enfatizando a importância de receber o resultado e ter um seguimento regular.

\section{ABSTRACT}

The increase in providing services, prevention and treatment of cancer in the uterine neck will be limited in their application, if women do not return to receive the result of the Papanicoulau exam. Not receiving the citopathological reports means discontinued treatment, which impedes efficient therapy for detected lesions. The objective of this study is to identify the reasons that cause women not to receive the results of the preventative exams. The study was carried out with women that had their prevented exam from the year 2005 through June 2006, and did not return to receive the result. A total of 29 women collaborated in the investigation. Factors that made it difficult to receive the results of the Papanicolau exam, according to the interviewed women range from questions related to the organization of the institution to questions related to the individual woman. However, the questions related to the institution represent a percentage much higher $(27.3 \%)$ in comparison with other questions. From factors that affect women not receiving the results, professionals can intervene in the hospital visit and emphasize the importance of obtaining the result and having regular follow-ups

\section{INTRODUCCIÓN}

A pesar de presentar el mayor potencial para la prevención y curación (alrededor del 100\%) cuando se diagnostica temprano, el cáncer de cuello uterino (CCU) es el segundo cáncer más común en las mujeres, lo que ha ocasionado más de 250.000 muertes en $2005,80 \%$ de estas muertes ocurrieron en países en desarrollo ${ }^{(1)}$.

El CCU es el cáncer más frecuente, sin considerar los tumores de piel no melanoma, en el norte del Brasil (22/100.000). En las regiones Sur (24/100.000), Centro-Oeste (19/100.000) y Nordeste (18/100.000) representa el segundo tumor más frecuente. En la región Sudeste el tercero $(18 / 100.000)^{(2)}$.

Este cáncer afecta raramente a las mujeres menores de 30 años, siendo más frecuente en mujeres mayores de 40 años. La causa principal de cáncer cervical de células escamosas es la infección por uno o más de los tipos oncogénicos conocidos (o de alto riesgo) del Virus del Papiloma Humano (VPH) ${ }^{(1)}$.

Los determinantes principales de la infección por VPH en hombres y mujeres están relacionados con el comportamiento sexual e incluyen la iniciación sexual temprana, el elevado número de parejas sexuales y las relaciones con parejas sexuales promiscuas. La infección por VPH de alto riesgo es más común en mujeres jóvenes y su prevalencia puede ser de hasta $25 \%$ a $30 \%$ en personas menores de 25 años ${ }^{(1)}$.

La historia natural del cáncer del cuello del útero se describe como una enfermedad iniciada con la transformación intra-epitelial que puede convertirse en una lesión de cáncer invasor en un plazo de 10 a 20 años. La evolución que se produce lentamente, pasa por un fase 
preclínica, asintomática, pero sus lesiones precursoras sólo se detectan mediante exámenes, y una fase sintomática, de acuerdo con la progresión de la enfermedad que se caracteriza por la presencia de secreción, sangrado vaginal irregular o después del acto sexual y dolor pélvico ${ }^{(3-4)}$.

Las estrategias de prevención secundaria al CCU consisten en el diagnóstico precoz de las lesiones del cuello uterino antes de que se hagan invasivas, de las técnicas de rastros 0 screening comprendidas por la colpocitología oncótica o examen de Papanicolaou, citología vaginal, colposcopía, cervicografía y, más recientemente, las pruebas de detección de ADN del VPH en frotis vaginal o histológico especímenes. El examen de Papanicolaou o citología vaginal, entre los métodos de detección, es considerado el más eficaz y eficiente para la aplicación de forma colectiva en los programas de detección de la UCC, siendo una técnica extendida más de 40 años ${ }^{(5)}$.

La baja cobertura del examen, la calidad en la recogida y la emisión de los informes citopatológicos, los factores relacionados con la baja adhesión de las mujeres al examen son aspectos relevantes en la problemática de la UCC. Sin embargo, dada la magnitud de este problema de salud pública y las posibilidades de curación de alta del CCU, el aumento en la prestación de servicios para la prevención y el tratamiento de los pacientes tendrán un margen limitado si estas mujeres no vuelven a recibir los resultados del examen ${ }^{(6)}$.

Estudios como los de Victor, Moreira e Araújo ${ }^{(6)}$ e o de Greenwood, Machado e Sampaio ${ }^{(7)}$ han abordado el no regreso de las mujeres para recibir el resultado del Papanicolau, con la pretensión de conocer las razones de por qué las mujeres no vuelven y el perfil de las mismas $^{(6-7)}$. Esto es problemático, ya que hay una inversión por el servicio en cada examen llevado a cabo. Ello afecta a los profesionales de alto nivel y personal técnico, así como otros gastos en el material, en el proceso de recolección de muestras, en la lectura de la lámina y en la impresión del resultado. Cuando la mujer no vuelve al servicio para recibir tal resultado, hay un desperdicio de tiempo y de recursos del servicio y de la mujer, pues el propósito del examen: la prevención del CCU, no es alcanzado.

Ante la experiencia en puestos de salud, se puede percibir la búsqueda activa de las mujeres por la atención ginecológica, aunque es necesario esperar muchos días para el examen. Por otra parte, después de la finalización de la colecta citológica, una parte de estas mujeres no vuelve al servicio para recibir los resultados, quedándo expuestas a graves enfermedades sin tratamiento adecuado. Dada esa realidad, la investigación tiene interés en conocer las razones por las que estas mujeres no vuelven para recibir el resultado preventivo.

\section{MATERIAL Y MÉTODOS}

Se trata de una investigación descriptiva y exploratoria, de naturaleza cuantitativa. La recolección de los datos fue llevada a cabo en una institución de salud: el Centro de Parto Lígia Barros Costa (CPNLBC), de la Universidad Federal del Ceará, en Fortaleza, Ceará, Brasil. En esta institución se realizan consultas de enfermería en el prenatal y consultas ginecológicas por estudiantes de enfermería bajo la supervisión de los profesores enfermeros.

La población del estudio estuvo integrado por mujeres que se sometieron al examen de prevención del cáncer cervicouterino en 2005 hasta junio de 2006 y no regresaron al servicio para recibir el resultado. Los datos fueron recogidos, y así se encontraron 101 mujeres en esta situación. 
Durante el estudio, se diseñó una estrategia para capturar la muestra inicialmente realizada por teléfono con un máximo de tres intentos para que las mujeres volviesen a la CPNLBC para recibir el resultado. Si no hubiese éxito después de tres intentos por teléfono, estaba prevista una visita a la casa para el mismo propósito. Las personas que no tenían teléfono también fueron visitadas. A estas mujeres se les garantizaba una nueva visita en un día específico para este fin (la obtención de resultados para las mujeres que incumplían) y si aceptaban, estaba disponible una nueva cosecha citológica en el momento de la consulta.

Durante su presencia en la institución, a la mujer se le pidió permiso para la participación en la investigación para hacer la aplicación de la herramienta de recolección de datos, una encuesta estructurada que constaba de los siguientes datos: identificación (nombre, edad, estado civil), resultados de la citología y microbiología y las razones del no recibimiento del examen de prevención del cáncer cervical.

Fueron establecidos los siguientes criterios para su inclusión en la muestra para mujeres mayores de 18 años o, en el caso de los menores, el tutor legal ha autorizado la participación en el estudio; llevado a cabo el examen en el período propuesto y no han recibido los exámenes citopatológicos; han sido contactadas por teléfono o visitas a domicilio y han venido al servicio para recibir el informe después del contacto y hacerse cargo del caso. Después de aplicar los criterios anteriores se obtuvo un total de 29 mujeres.

Los datos se recogieron entre los meses de octubre y diciembre de 2006, organizados en el sistema Statistical Package for the Social Sciences (SPSS) y presentados en tablas analizadas a través de la literatura.

Se garantizó a las personas que se dispusieron a participar del estudio, el sigilo de identidad, según los principios descritos en la Resolución no196/96 que trata de investigación que abarca seres humanos. El proyecto fue aprobado por el Comité de Ética

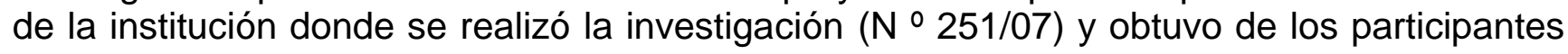
la firma en el Término de Consentimiento Informado.

\section{RESULTADOS Y DISCUSIÓN}

De las 645 mujeres referidas a partir de enero de 2005 hasta junio de 2006, 101 no se presentaron al servicio para recibir los resultados de los exámenes de prevención, lo que representa $15,6 \%$ de las mujeres. De las 101 mujeres, sólo $29(28,7 \%)$ regresaron al servicio para recibir el resultado después de fijar un horario para el regreso por teléfono 0 visita en el domicilio. Se ha encontrado dificultad para fijar un horario para los regresos, ya que algunas no tenían teléfono y la dirección no era la misma que había en el informe.

Este dato señala la importancia de realizar un registro completo, comprobando todas las informaciones con el cliente, ya que la dirección y el número de teléfono se registran con el fin de localizar caso no se presente a la consulta de regreso o cuando el resultado del examen requiere intervención inmediata. Este resultado es importante para la evaluación del servicio, porque de él se ha reflexionado sobre la importancia del registro con los estudiantes de enfermería y enfermeros del servicio.

Entre las funciones de los Agentes Comunitarios de Salud (ACS) está el seguimiento de las mujeres que presentaron el resultado del examen preventivo alterado, muestras insatisfactorias y sin anormalidades para el seguimiento regular ${ }^{(8)}$. En el servicio en cuestión, no había ACS, lo que ha impedido la realización de esta acción. Como había 
pocos estudiantes de enfermería, las visitas domiciliarias se limitaron a las puérperas y así se procedió a la búsqueda activa a través de llamadas telefónicas.

Las pacientes que contestaron al teléfono y no se presentaron al servicio en la fecha prevista, recibieron un nuevo contacto para citarse, sin embargo, algunas señalaron que no tenían ningún interés en esta nueva consulta para obtener el resultado o porque no deseaban o por ya han llevado a cabo un nuevo examen en otro servicio de salud.

El grupo de edad con mayor número de mujeres ausentes $(65,5 \%)$ en este estudio se queda entre 16 y 37 años de edad. En el estudio de Victor, Moreira e Araújo ${ }^{(6)}$, el grupo de edad de las mujeres menores de 34 años representaron el mayor porcentaje de exámenes de Papanicolaou que no fueron devueltos ${ }^{(6)}$. Aunque el cáncer concierne a las mujeres mayores de 30 años en mayor proporción, la prevención debe iniciarse de forma precoz entre las jóvenes. Así pues, para que el examen alcance su finalidad, es necesario que todas las mujeres vuelvan para recibir el resultado.

Aunque sólo nueve (31\%) mujeres de 37-59 no regresaron para recibir el resultado, esto dato es importante ya que la literatura revela que el mayor número de casos de CCU ocurre entre 35 y 59 años $^{(9)}$.

Después de la recopilación de los diagnósticos de enfermería más frecuentes en la atención a la mujer llevados a cabo en una UBS, fueron identificados 60 diagnósticos, de los cuales uno de los cinco más frecuentes fue el escaso conocimiento sobre la finalidad, la importancia y frecuencia del examen de Papanicolaou, los materiales necesarios para la realización y la necesidad de volver a la institución donde realizó el examen preventivo para conocer el resultado. Otro diagnóstico señalado fue el riesgo para la infección con los siguientes factores de riesgo: actividad sexual asociada a no realización del examen de Papanicolaou o a su aplicación durante más de dos años y la falta de conocimiento sobre la importancia de volver a la institución para la evaluación del resultado ${ }^{(9)}$.

Es necesario invertir en un enfoque más humano para la realización del examen preventivo a fin de aclarar a las mujeres sobre todo el proceso, desde la entrevista y el examen físico, la recogida de materiales y las orientaciones finales. La explicación sobre el examen de manera más natural facilita la comprensión del proceso sin la construcción de opiniones negativas sobre el mismo ${ }^{(10)}$.

El enfermero, como profesional del equipo de salud, que desempeña sus actividades como colaborador en la prevención del cáncer de cuello uterino, debe dirigirse a las mujeres durante la consulta de enfermería sobre la importancia de la realización del examen de Papanicolau, desde la colecta citológica hasta la búsqueda del resultados ${ }^{(8)}$.

En relación a las afecciones registradas en los resultados de los exámenes, se señaló que $29(100 \%)$ mujeres (tabla 1) poseían la presencia del proceso inflamatorio.

Toda manifestación de la inflamación y/o infección del tracto genital femenino inferior, es decir, de la vulva, de la vagina y del epitelio escamoso del cuello uterino (exocérvix), se llama vulvovaginitis. El cuadro clínico varía de acuerdo con la etiología, puede manifestarse por la presencia de flujo vaginal, cuyas características pueden variar notablemente, lo cual puede haber variado la coloración (blanco, amarillo, gris, verde), tener o no olor 
desagradable, dolor, irritación, picazón o ardor en la vagina o en la vulva, dolor o ardor al orinar y molestias pélvicas. Cabe señalar que estos signos y síntomas son inespecíficos, además, muchas infecciones genitales pueden ser completamente asintomáticas ${ }^{(8)}$.

Tabla 1 - Distribución de las mujeres mientras al nivel del proceso inflamatorio y microbiología presentados en los laudos. Fortaleza, Ceará, Brasil, 2007

\begin{tabular}{lll} 
Variables & N & $\%$ \\
\hline Inflamación & & \\
Leve & 10 & 34,5 \\
Moderada & 15 & 51,7 \\
Marcada & 04 & 13,8 \\
Microbiología & & \\
Lactobacillus sp & 10 & 34,5 \\
Gardnerella & 08 & 27,5 \\
vaginallis/Mobiluncus & & \\
Tricomoniasis vulvovaginal & 01 & 3,4 \\
Cocos, Bacilos & 05 & 17,2 \\
Candida albicans & 04 & 13,7 \\
Flora bacteriana escasa & 01 & 3,4 \\
Total & 29 & 100,0 \\
\hline
\end{tabular}

La vulvovaginitis es uno de los problemas ginecológicos más comunes y molestos que afectan la salud de las mujeres y representa aproximadamente $70 \%$ de las reclamaciones en consultas de ginecología(8). Teniendo en cuenta que estas mujeres deberían orientarse durante la visita de seguimiento sobre los posibles factores físicos, químicos, hormonales, anatómicos y biológicos relacionados con la aparición de la vulvovaginitis, esta posibilidad se ha perdido porque estas mujeres no regresan a la unidad de salud ${ }^{(6)}$.

Las formas más comunes de vulvovaginitis son: candidiasis vulvovaginal causada por la candida albicans, la vaginosis bacteriana causada por Gardnerella vaginalis y tricomoniasis vulvovaginal causada por trichomonas vaginalis.

Los resultados microbiológicos compatibles con Lactobacillus sp (34,5\%), cocos y bacilos $(17,2 \%)$ son considerados hallazgos normales. Son parte de la flora vaginal y no caracterizan infecciones que requieren tratamiento ${ }^{(11)}$.

La Gardnerella vaginallis se encontró en $17,2 \%$ de los informes, es una bacteria que se encuentra en bajas concentraciones en la flora vaginal sin causar daño. Sin embargo, algunos factores pueden desencadenar el proceso inflamatorio, cambiando el equilibrio biológico por la prevalencia de esta bacteria. A este cuadro se ha convenido en llamar de vaginosis bacteriana ${ }^{(12)}$. No se trata de una infección de transmisión sexual, solo puede ser desencadenada por el acto sexual en mujeres predispuestas, al tener contacto con el semen, el cual tiene un alto $\mathrm{pH}^{(8)}$.

Una de las características clínicas de la vaginosis bacteriana es el flujo vaginal blanco, gris, de aspecto cremoso o líquido, a veces bulloso, con mal olor, más agudo después de una relación y durante la menstruación; dolor después de las relaciones sexuales (poco frecuentes) $)^{(8)}$. 
La Candida albicans es un hongo oportunista que vive como comensal en la mucosa del aparato digestivo, se encuentra en la vagina en $30 \%$ de las mujeres sanas o asintomática, forma parte de la flora vaginal normal y se puede identificar en las mujeres premenopáusicas o vírgenes. Se observa un aumento en la frecuencia de la candidiasis, conocida como vulvovaginitis, debido al inicio de la actividad sexual regular ${ }^{(12)}$. La Candida albicans fue presentada en $13,7 \%$ de los registros analizados.

Los signos y síntomas de la candidiasis dependen del grado de la infección y de la localización del tejido inflamado, puede estar presente por separado o conjuntamente, e incluyen: picazón vulvovaginal, ardor o dolor al orinar, flujo vaginal blanco, grumoso, sin olor y con aspecto casposo, hiperemia, edema vulvar, fisuras y maceración de la vulva, vagina y cuello uterino y cubierto con placas blancas o blanco grisáceo, adheridos a la mucosa, y dolor durante el acto sexual ${ }^{(8)}$.

Trichomonas vaginalis es el agente causal de la forma más común de enfermedad parasitaria, enfermedad sexualmente transmitida (ETS). Trichomonas vaginalis tiene que superar distintos obstáculos y respuesta inmunes del huésped para colonizar la mucosa y establecer la infección ${ }^{(12)}$.

La mera constatación de Trichomonas vaginalis en una citología de rutina requiere el tratamiento de la mujer y también de la pareja sexual, ya que se trata de una enfermedad de transmisión sexual. También se deben realizar las siguientes medidas complementarias: asesoramiento, ofrecimentos de pruebas de VDRL, HIV, serología para la hepatitis B y C. Debe además enfocarse la adherencia al tratamiento, notificar y fijar el regreso ${ }^{(8)}$.

La tricomoniasis vaginal puede afectar el resultado de la citología. Por lo tanto, en los casos en que no hay cambios morfológicos de células, se debe realizar el tratamiento y la repetición de la citología con tres meses para evaluar si hay persistencia de estos cambios.

Teniendo en cuenta todos los daños que cada una de estas infecciones pueden causar, el no regreso de aquellas mujeres que presentan informe con tricomoniasis, vaginosis bacteriana y candidiasis dificulta el acceso de las mujeres a un tratamiento adecuado y a las información sobre cómo evitarlas.

La estructura organizativa de la propia institución puede servir como una barrera para que el resultado del examen sea devuelto a las mujeres. En esta investigación, los factores relacionados con la institución fueron: los informes no estaban listos $(27,3 \%)$, el puesto estaba cerrado $(5,5 \%)$ y el profesional del servicio dijo que el examen ya no estaba $(1,8 \%)$.

El hecho de que los informes no se quedaran listos en la fecha establecida revela la importancia de que los profesionales del servicio de salud encaminen pronto las láminas al laboratorio, para que los patólogos agilicen la lectura de ellas.

El servicio de salud en cuestión no dispone de su propio laboratorio para el análisis de las láminas, y así dirige todas los exámenes realizados durante la semana, en viernes, para el laboratorio de un hospital escuela de referencia en Fortaleza, Brasil. Debido a este desplazamiento, hay un mayor retraso hasta que los resultados están disponibles dentro de la institución en cuestión.

Investigación llevada a cabo en una Unidad Básica de Salud (UBS) en Fortaleza sobre el no regreso de las mujeres para recibir los resultados del examen de prevención del CCU ha identificado algunos obstáculos en la dinámica del servicio, lo que dificultaba el acceso de la mujer a la consulta de seguimiento, como por el ejemplo: el número de mujeres que realizan 
la recogida citológica era el doble de las fichas distribuidas para la atención en el regreso, quedándose, por lo tanto, $50 \%$ de las mujeres sin garantía de continuidad de la atención. Además, no había, en la unidad, la búsqueda activa de mujeres examinadas debido a los resultados que necesitaban de la intervención o encaminamiento con mayor urgencia, como en los casos de carcinoma in situ ${ }^{(6)}$.

Tabla 2 - Distribución de las mujeres según los motivos que las llevaron a no recibir el informe citopatológico. Fortaleza, Ceará, Brasil, 2007

\begin{tabular}{llc} 
Los Motivos & $N$ & $\%$ \\
\hline El informe no estaba listo & 15 & 27,3 \\
Trabajo durante el funcionamiento del servicio de salud & 10 & 18,2 \\
Falta de tiempo & 4 & 7,3 \\
Abandono & 4 & 7,3 \\
Viajes & 4 & 7,3 \\
Olvido & 4 & 7,3 \\
Servicio de salud cerrado & 3 & 5,5 \\
Falta de interes & 3 & 5,5 \\
Motivos personales y/o familiares & 3 & 5,5 \\
Debido al tiempo, se pensaba que el laudo ya no estaba en el & 1 & 1,8 \\
servicio de salud & 1 & 1,8 \\
Acomodación & 1 & 1,8 \\
Larga distancia del sitio al servicio de salud & 1 & 1,8 \\
Pérdida de la tarjeta para recibir el resultado & 1 & 1,8 \\
La no validación del resultado, según el profesional del servicio & 55 & 100, \\
Total & & 0 \\
\hline
\end{tabular}

${ }^{*}$ Hubo más de una respuesta por mujer.

Otra razón muy importante relatada por las mujeres para la no recepción de los informes fue el hecho de estar trabajando en las horas de funcionamiento del puesto. Hay que recordar que un proyecto aprobado en 2000 por la Cámara de Diputados, y encaminado al Congreso Brasileño, garantiza el derecho a un día por año para que toda empleada de la empresa pública o privada haga el examen de Papanicolaou y hace obligatoria la prevención del cáncer ginecológico para la empleada que se incorpore al servicio público o privado ${ }^{(13)}$.

En otra investigación, también llevada a cabo en una UBS de Fortaleza, con el mismo tema, las mujeres citaron como obstáculos para no regresar a la unidad: la situación de trabajo de la mujer, las dificultades financieras y la movilidad, la falta de directrices adecuadas para parte de los profesionales sobre la necesidad de volver, las dificultades para trasladar la fecha de retorno, y largos tiempos de espera para fijar un horario para la consulta y la reducción de los recursos materiales y humanos. Estos factores, según ellas, les impiden continuar el proceso en busca de prácticas preventivas ${ }^{(7)}$.

Por otra parte, algunas mujeres del mismo estudio, así como las de este otro, presentaron justificaciones perfectamente delimitadas para la no asistencia a la nueva consulta, e incluso, para falta de búsqueda de cualquier otra alternativa. Este comportamiento nos lleva a cuestionar la importancia dada por estas mujeres al examen, considerando que la consulta de regreso es programada con un mes de antelación, lo que permite a la mujer planificar adecuadamente su regreso al servicio ${ }^{(7)}$. 
Es fundamental cambiar el cuadro de la cobertura de los exámenes en relación a las mujeres brasileñas que aún carecen de acceso al mismo, sin embargo, hay que pensar primero primero en la calidad y estructura de los servicios que ofrece el examen hoy, que no proporcionan una atención integral a las mujeres con el fin de luchar eficazmente contra el CCU.

Los estudios sobre este tema de no retorno de las mujeres para recibir el resultado del examen de Papanicolau han puesto de manifiesto una triste realidad de los servicios de atención primaria de salud en relación al aspecto organizativo de la unidad con el fin de permitir el seguimiento de las mujeres que buscan el Papanicolaou, lo que trae como enfoque para la discusión sobre la falta de información proporcionada por los profesionales sobre el examen. El hecho es que la desinformación o la información insuficiente proporciona un entorno adecuado para que las mujeres no lleven a cabo el examen preventivo, y para las que desempeñan el no regreso.

Después de la recopilación de los diagnósticos de enfermería más frecuentes en la atención a las mujeres realizada en una UBS, fueron identificados 60 diagnósticos, de los cuales uno de los cinco más frecuentes fue el escaso conocimiento relacionado con la finalidad, la importancia y frecuencia del examen de Papanicolaou, los materiales necesarios para su realización y la necesidad de volver a la institución donde se realizó el examen para conocer el resultado. Otro diagnóstico que se encontró fue el riesgo para la infección presentando los siguientes factores de riesgo: actividad sexual activa asociada con la no realización del examen de Papanicolaou o de su aplicación durante más de dos años, y la falta de conocimiento sobre la importancia de volver a la institución para la evaluación de resultados $^{(9)}$.

La aparente falta de interés de la mujer para obtener el resultado no siempre es unilateral. A veces no es ofrecida por el servicio orientación clara sobre la importancia de volver para la validez de la recolecta del examen. El hecho de la paciente de no recibir el examen o la manera como él es comunicado, si personalmente o no, puede representar una oportunidad perdida para deshacer las creencias y las actitudes negativas en cuanto al examen, a su propósito, a la importancia de sus resultados y al propio cáncer de cuello uterino ${ }^{(5)}$.

El momento en que una mujer encuentra el profesional de salud es de importancia singular. Es esencial que los profesionales proporcionen informaciones relevantes acerca de la importancia de la realización del examen para la prevención del cáncer cervical y su seguimiento.

\section{CONCLUSIONES}

Mucho se ha investigado sobre la CCU en el mundo, sin embargo, la mayoría de estos estudios se han ocupado de la prevención del cáncer de la UCC desde el punto de vista técnico, mientras que las cuestiones sociales, culturales y las características de los servicios de salud no se consideran, o en todo caso de modo muy simplista. Las condiciones en que se han llevado a cabo estos estudios no reproducen las características generales de la población y de los servicios de salud en los países en desarrollo.

Con esta investigación se han hallado los factores que dificultan la recepción del resultado del examen de Papanicoloau que van desde las cuestiones relativas a la organización de la institución hasta los asuntos relacionados con la propia mujer. Sin embargo, las cuestiones relativas a la institución representan una proporción mucho mayor en comparación con las demás. 
A pesar de la pequeña muestra del estudio, otros estudios han demostrado que la realidad es la misma. Es preciso reflexionar sobre la calidad de la atención en los servicios a las mujeres que realizan este examen, en la perspectiva de un trabajo en salud más humanizado para proporcionar a las mujeres un entorno propicio para la promoción de salud. Una de las actividades del programa de control de cáncer cervicouterino es la educación para la salud. Así, según el Ministerio de Salud, ninguna acción de control del cáncer cervicouterino alcanzará sin la participación del componente educativo que llega a la población de las mujeres y a los profesionales de la salud. Se deben ofrecer a todas las mujeres que buscan los servicios de salud, por la razón que sea, acciones individuales o en grupos de reflexión sobre los beneficios que se derivan de estas actividades.

El problema relacionado con el no regreso de las mujeres para recibir el resultado de la citología tiene múltiples factoress, sin embargo, pero se asegura que la educación alcanza todas estas áreas y que si se lleva a cabo de manera dialógica y reflexiva, conducirá a las mujeres de la pasividad a la criticidad, de manera que se cambien en sujetos de proceso de adopción de comportamientos saludables y de la búsqueda de mejores servicios para prevenir el CCU.

No se justifica el elevado número de muertes de mujeres debido a una enfermedad cuya prevención se presenta tan efectiva cuando se aplica adecuadamente. Pero, al analizar las estadísticas de los casos, es necesario considerar que la prevención no debe ser una condición a planificar, organizar en un caso aislado y separado del contexto social. La prevención implica las políticas públicas, las acciones y la participación profesional. Estas acciones coordinadas se traduciran en beneficios para los usuarios del sistema de salud, al considerar estas variables de modo que puedan ser trabajadas con eficacia.

\section{REFERENCIAS}

(1) Organización Mundial de la Salud. Control integral del cáncer cervicouterino: guía de práticas esenciales. Genebra: Organización Mundial de la Salud; 2007.

(2) Ministério da Saúde (BR). Secretaria de Atenção à Saúde. Instituto Nacional de Câncer. Coordenação de Prevenção e Vigilância de Câncer. Estimativas 2008: Incidência de Câncer no Brasil. Rio de Janeiro: INCA; 2007.

(3) Ministério da Saúde (BR). Secretaria Nacional de Assistência à Saúde. Instituto Nacional do Câncer. Coordenação de Prevenção e Vigilância. Falando sobre câncer do colo do útero. Rio de Janeiro: MS/ INCA; 2002.

(4) Smeltzer SC, Bare BG, organizadoras. Tratado de enfermagem médico-cirúrgico. 9a ed. Rio de Janeiro: Guanabara Koogan; 2002.

(5) Pinho AA, França-Júnior I. Prevenção do câncer de colo o útero: um modelo teórico para analisar o acesso e a utilização do teste de Papanicolaou. Rev Bras Saúde Mater Infant. 2003; 3(1):34-8.

(6) Victor JF, Moreira TMM, Araújo AR. Exames de prevenção de câncer de colo uterino realizados e não retirados de uma Unidade Básica de Fortaleza - Ceará. Acta Paul Enf. 2004; 17(4):407-11.

(7) Greenwood AS, Machado MFAS, Sampaio NVV. Motivos que levam as mulheres a não retornarem para receber o resultado do exame de Papanicolaou. Rev Latino-Am Enfermagem. 2006; 14(4):503-9.

(8) Ministério da Saúde (BR). Secretaria de Atenção à Saúde. Departamento de Atenção Básica. Controle dos cânceres do colo do útero e da mama: Brasília: Ministério da Saúde; 2006. 
(9) Gerk MAS, Barros SMO. Intervenções de enfermagem para os diagnósticos de enfermagem mais freqüentes em dois serviços públicos de assistência à saúde da mulher. Acta Paul Enferm. 2005; 18(3):260-8.

(10) Lucarini ACBS, Campos CJG. The search for the accomplishment of the preventive examination of cancer cytology: a qualitative study. Online Braz J Nurse. [Online]. 2007 January; 6(0). [citado em 2007 mar 14]. Disponível em http://www.uff.br/objnursing/index.php/nursing/article/view/671/156.

(11) Ministério da Saúde (BR). Instituto Nacional do Câncer. Nomenclatura brasileira para laudos cervicais e condutas preconizadas: recomendações para profissionais de saúde. $2^{\mathrm{a}}$ ed. Rio de Janeiro: INCA; 2006.

(12) Passos MRL. Deessetologia: DST. 5ª̣ed. Rio de janeiro: Cultura Médica; 2005.

(13) Agência Estado. Projeto dá um dia para o Papanicolau[Internet]. São Paulo: Agência Estado.

[citado 2008 fev 02]. Disponível http://www.estadao.com.br/agestado/nacional/2000/out/04/286.htm. 